\title{
What Limits the Maximum Output Power of Long-Wavelength AlGaInAs/InP Laser Diodes?
}

\author{
Joachim Piprek, Senior Member, IEEE, J. Kenton White, Member, IEEE, and Anthony J. SpringThorpe
}

\begin{abstract}
We analyze the high-temperature continuous-wave performance of $1.3-\mu \mathrm{m}$ AlGaInAs/InP laser diodes grown by digital alloy molecular-beam epitaxy. Commercial laser software is utilized that self-consistently combines quantum-well bandstructure and gain calculations with two-dimensional simulations of carrier transport, wave guiding, and heat flow. Excellent agreement between simulation and measurements is obtained by careful adjustment of material parameters in the model. Joule heating is shown to be the main heat source; quantum-well recombination heat is almost compensated for by Thomson cooling. Auger recombination is the main carrier loss mechanism at lower injection current. Vertical electron escape into the $p$-doped InP cladding dominates at higher current and causes the thermal power roll-off. Self-heating and optical gain reduction are the triggering mechanisms behind the leakage escalation. Laser design variation is shown to allow for a significant increase in the maximum output power at high temperatures.
\end{abstract}

Index Terms-Laser thermal factors, optoelectronic devices, quantum-well devices, semiconductor device modeling, semiconductor laser.

\section{INTRODUCTION}

A $\mathrm{T}$ 1.3- $\mu \mathrm{m}$ wavelength, AlGaInAs/InP laser diodes have shown better performance than conventional InGaAsP/InP lasers, enabling uncooled continuous-wave (CW) operation at high ambient temperatures [1]. This is mainly attributed to the larger conduction band offset which reduces the electron leakage from the multi-quantum-well (MQW) active region. However, output power and linearity are limited by the roll-off of the light-current $(L-I)$ characteristic. The $L-I$ roll-off is typical for laser diodes and it is attributed to self-heating of the device in $\mathrm{CW}$ operation. Temperature elevation affects a number of key physical mechanisms in laser diodes, including the optical gain, optical losses, and nonradiative recombination mechanisms [2]. It also reduces the bandgap and the carrier mobility.

Based on measured laser characteristics, we analyze the interaction of these internal processes to evaluate the detailed physical mechanisms that lead to the power roll-off. Advanced laser simulation is used as an analysis tool. First, the model parameters are calibrated so that measurements at different temperatures can be reproduced by the simulation simultaneously (Section III). Second, based on this model validation, the internal

Manuscript received January 11, 2002; revised May 21, 2002.

J. Piprek is with the Electrical and Computer Engineering Department, University of California, Santa Barbara, CA 93106 USA.

J. K. White and A. J. SpringThorpe are with Nortel Networks, Ottawa, ON K2H 8E9, Canada.

Publisher Item Identifier 10.1109/JQE.2002.802441.

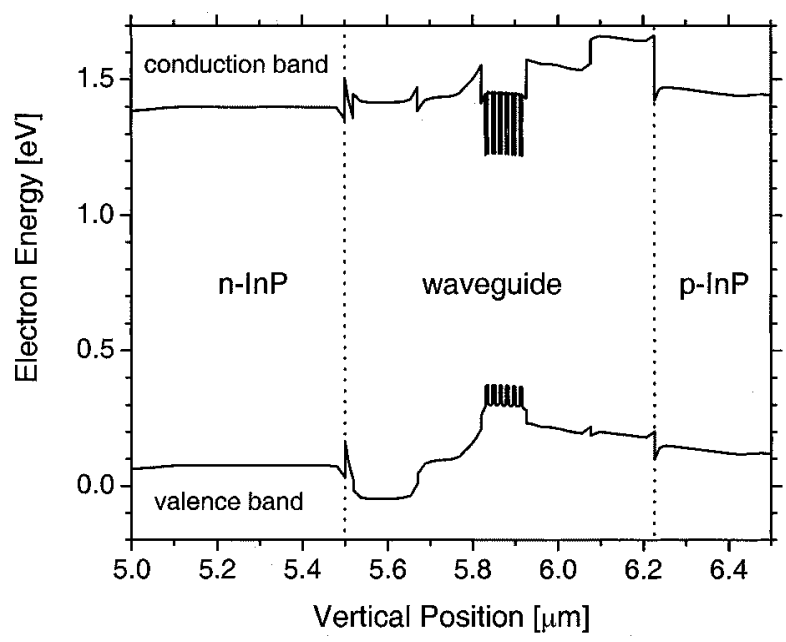

Fig. 1. Energy band diagram near threshold $\left(T=25^{\circ} \mathrm{C}\right)$.

physical processes are analyzed that affect the power roll-off in $\mathrm{CW}$ operation at high temperatures (Section IV). Triggered by gain reduction and subsequent increase in MQW carrier density, electron leakage is found to be the main reason for the power roll-off. Finally, Section V evaluates several device variations that aim at higher maximum output power.

\section{Device Structure, Fabrication, AND Measurements}

The MQW active region contains six 6-nm-thick $\mathrm{Al}_{0.175} \mathrm{Ga}_{0.095} \mathrm{In}_{0.73} \mathrm{As}$ quantum wells $(1.4 \%$ compressive strain) with a measured photoluminescence (PL) peak near $1.3 \mu \mathrm{m}$. The seven 10-nm-thick $\mathrm{Al}_{0.27} \mathrm{Ga}_{0.21} \mathrm{In}_{0.52}$ As barriers are unstrained. The undoped MQW is sandwiched between two $\mathrm{Al}_{0.40} \mathrm{Ga}_{0.08} \mathrm{In}_{0.52} \mathrm{As}$ and two AlInAs confinement layers. Each of the four layers is 150 -nm thick. This waveguide region is grown on $\mathrm{n}$-InP and 20 -nm n-doped $\mathrm{Al}_{0.40} \mathrm{Ga}_{0.08} \mathrm{In}_{0.52} \mathrm{As}$ and is covered by p-doped InP. The last $1600 \mathrm{~nm}$ of p-InP form the ridge waveguide (width $W=2 \mu \mathrm{m}$ ). The ridge is concluded by $200 \mathrm{~nm}$ of $\mathrm{p}$-InGaAs. The semiconductor structure is grown by digital alloy molecular-beam-epitaxy (MBE). Fabry-Perot lasers are formed with cleaved facets and $L=700 \mu \mathrm{m}$ cavity length. Further details of the fabrication process are described elsewhere [3]. The energy band diagram at the vertical laser axis is shown in Fig. 1.

$L-I$ and current-voltage $(I-V)$ characteristics are measured in $\mathrm{CW}$ operation at different ambient temperatures which shall be used in the next section to calibrate the laser model. In addition, pulsed measurements on broad-area lasers $(W=50 \mu \mathrm{m})$ with different cavity length are employed. 


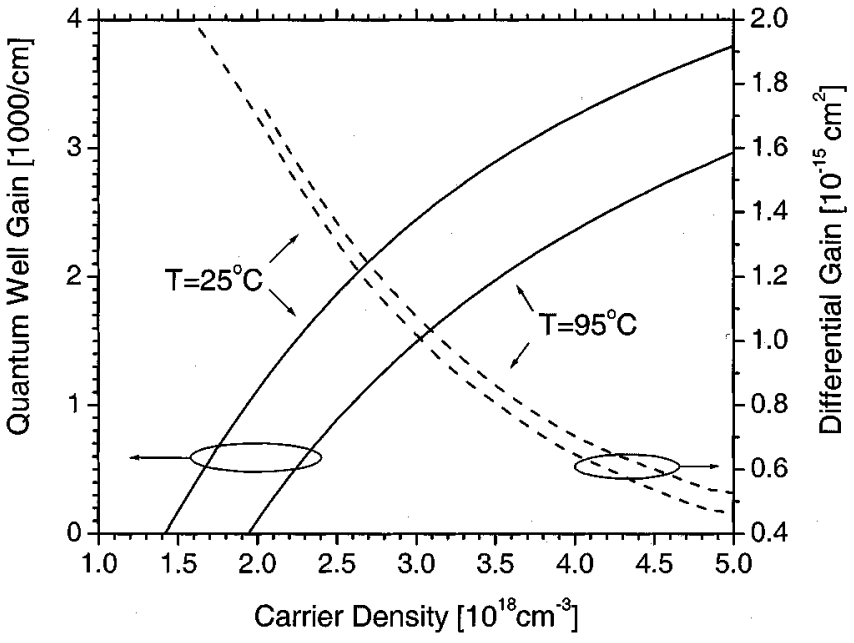

Fig. 2. Optical gain (solid) and differential gain (dashed) versus carrier density.

\section{LASER Model AND Material PARAMETERS}

Our analysis utilizes a commercial two-dimensional (2-D) laser simulation tool. ${ }^{1}$ The software self-consistently combines 2-D carrier transport, heat flux, optical gain computation, and wave guiding within the transversal plane $(x, y) .{ }^{2}$ The same software was employed previously to investigate temperature effects in pulsed laser operation, showing excellent agreement with measurements [2]. Here, we discuss those aspects of the model that are crucial to our $\mathrm{CW}$ analysis. The model requires a large number of material parameters which need to be adjusted before a reliable analysis and optimization of the device can be performed. This calibration process consists of several stages and it involves different measurements.

Gain calculations are the most important part of any laser simulation. The gain model is based on $4 \times 4 \mathrm{kp}$ band structure calculations, including valence-mixing effects [4]. The calculated quantum-well strain of $1.37 \%$ is in good agreement with experimental results. A Lorentz broadening function with $\tau_{\mathrm{sc}}=0.06$ ps scattering time is employed in the gain calculation [5]. Fig. 2 shows the corresponding optical gain. At room temperature, the transparency density is about $1.4 \times 10^{18} \mathrm{~cm}^{-3}$ and the initial differential gain is $d g / d N=2 \times 10^{-15} \mathrm{~cm}^{2}$. The gain peaks near $1.3 \mu \mathrm{m}$, which is in good agreement with the measured lasing wavelength. This indicates that the bandgap of the strained quantum well is calculated correctly.

The drift-diffusion model of carrier transport considers Fermi statistics and thermionic emission at hetero-barriers. Thermionic emission is mainly controlled by the offset of the conduction band $\left(\Delta E_{c}\right)$ and the valence band $\left(\Delta E_{v}\right)$. For the AlGaInAs material system, a band offset ratio of $\Delta E_{c} / \Delta E_{g}=0.72$ is commonly assumed. The energy band diagram in Fig. 1 illustrates the large conduction band offset of $\Delta E_{c}=0.225 \mathrm{eV}$ within the MQW. The valence band offset is $\Delta E_{v}=0.091 \mathrm{eV}$ for heavy holes and only $2 \mathrm{meV}$ for light holes, due to the compressive strain within the quantum wells. AlInAs and InP exhibit a type-II interface with $\Delta E_{v}=0.1 \mathrm{eV}$.

${ }^{1}$ LASTIP by Crosslight Software.

${ }^{2}$ Details of the Crosslight Software Inc. laser model are available at: http://www.crosslight.com.

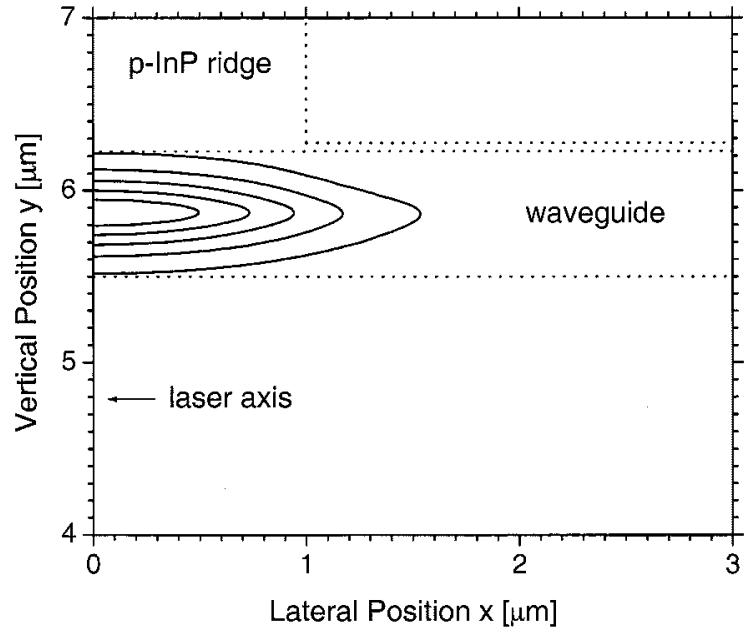

Fig. 3. Fundamental optical mode.

TABLE I

ROOM-Temperature VAlues FOR Key MATeRial Parameters ( $n_{r}$-REFRACTIVE INDEX AT $1.3-\mu \mathrm{m}$ WAVELENGTH, $k_{p}$ - HOLE ABSORPTION PARAMETER, $\mu_{\text {po }}$-HOLE MOBILITY, $\kappa$-THERMAL CONDUCTIVITY)

\begin{tabular}{l|l|l|l|l}
\hline symbol & $\mathrm{n}_{\mathrm{r}}$ & $\mathrm{k}_{\mathrm{p}}$ & $\mu_{\mathrm{po}}$ & $\kappa$ \\
\hline unit & & $\mathrm{m}^{2}$ & $\mathrm{~m}^{2} / \mathrm{Vs}$ & $\mathrm{W} / \mathrm{K} \mathrm{m}$ \\
\hline $\mathrm{InP}$ & 3.2 & $13 \times 10^{-22}$ & 0.02 & 68 \\
\hline $\mathrm{Al}_{0.4} \mathrm{Ga}_{0.08} \mathrm{In}_{0.52} \mathrm{As}$ & 3.34 & $13 \times 10^{-22}$ & 0.005 & 4 \\
\hline $\mathrm{AlIn} \mathrm{As}$ & 3.29 & $13 \times 10^{-22}$ & 0.005 & 5 \\
\hline $\mathrm{Al}_{0.27} \mathrm{Ga}_{0.21} \mathrm{In}_{0.52} \mathrm{As}$ (barr.) & 3.43 & $13 \times 10^{-22}$ & 0.005 & 4 \\
\hline $\mathrm{Al}_{0.175} \mathrm{Ga}_{0.095} \mathrm{In}_{0.73} \mathrm{As}(\mathrm{QW})$ & 3.75 & $30 \times 10^{-22}$ & 0.005 & 4 \\
\hline
\end{tabular}

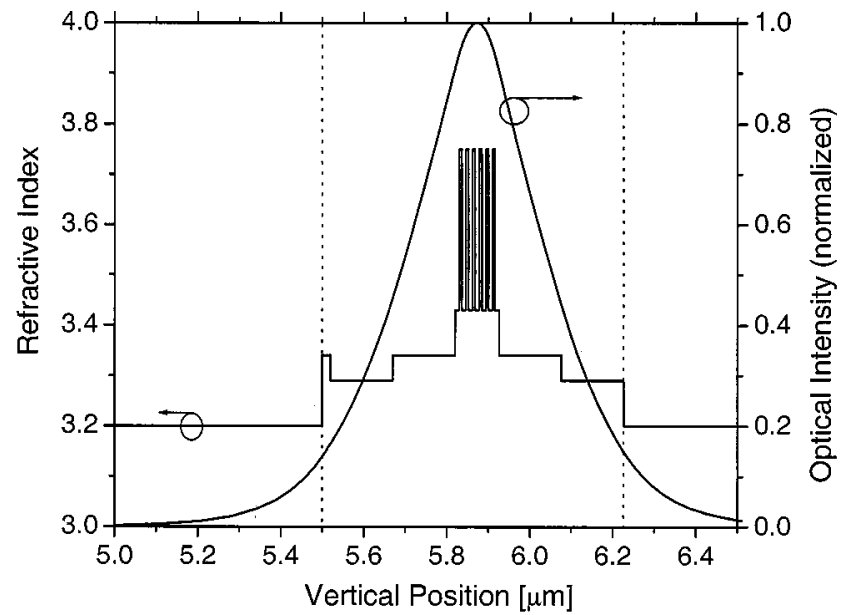

Fig. 4. Vertical profile of refractive index and optical wave.

The fundamental optical mode is shown in Fig. 3 and it is calculated using the effective index approximation which requires accurate refractive index values for each material. The popular Sellmeier formulas for the refractive index [6] are often inaccurate for photon energies near the bandgap. Instead, we employ the Adachi model which was developed for energies close to the bandgap [7], [8]. Numerical values are listed in Table I. The vertical refractive index profile of our device is plotted in Fig. 4 as well as the normalized optical wave profile in the vertical direction (cold cavity). The optical confinement factor is $\Gamma=0.083$. 


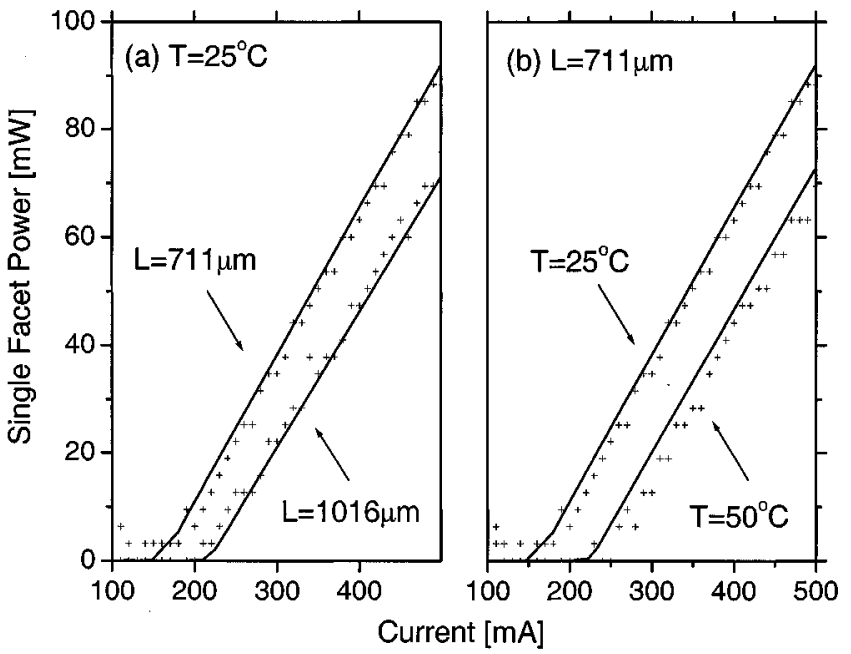

Fig. 5. Simulated (lines) and measured $(+) L-I$ characteristics in pulsed operation for: (a) different cavity length $L$ and (b) different ambient temperature $T(W=50 \mu \mathrm{m})$.

In our laser, intervalence band absorption (IVBA) and Auger recombination are considered main loss mechanisms for photons and electrons, respectively. $L-I$ characteristics measured on broad-area devices $(W=50 \mu \mathrm{m})$ in pulsed operation are employed to adjust the Auger coefficient and internal optical loss parameters in the laser model. Both these loss parameters need to be balanced to achieve simultaneous agreement with the measured threshold current and slope efficiency [9]. Short current pulses are used to avoid any significant self-heating of the device. In this case, heat flux is excluded from the model and the broad-area device is considered one-dimensional (1-D). The resulting $L-I$ fit is plotted in Fig. 5 for two different cavity lengths $L$ and for two different ambient temperatures $T$.

Internal optical loss in 1.3- $\mu \mathrm{m}$ lasers is expected to be dominated by IVBA, which is proportional to the local hole density $p(x, y)$. In our model, the local absorption is calculated as

$$
\alpha(x, y)=\alpha_{s}+k_{p} p(x, y)
$$

with a constant scattering loss $\alpha_{s}$ and $k_{p}=13 \times 10^{-18} \mathrm{~cm}^{2}$ for bulk material (Table I) [10]. For quantum wells, $k_{p}$ is difficult to measure and no reliable experimental results are published. In addition, this parameter acts as a correction of the QW gain which also rises almost linearly with the carrier density. Thus, by using $k_{p}$ as a fit parameter within the quantum wells, inaccuracies of the gain calculation are leveled. Eventually, we obtain the best agreement with the $L-I$ measurements in Fig. 5 using the QW parameter $k_{p}=30 \times 10^{-18} \mathrm{~cm}^{2}$. Thereby, the effective differential gain is slightly reduced by $0.03 \times 10^{-15} \mathrm{~cm}^{2}$ (cf. Fig. 2). For our broad-area devices ( $W=50 \mu \mathrm{m}$ and $L=$ $711 \mu \mathrm{m})$, the modal loss parameter becomes $\alpha_{i}=1.48 \mathrm{~cm}^{-1}$ at room temperature and $1.61 \mathrm{~cm}^{-1}$ at $T=50^{\circ} \mathrm{C}$. Scattering losses at the side wall of the ridge are negligible for broad-area devices $\left(\alpha_{s}=0\right)$. A facet reflectance of $R=0.29$ is assumed.

The Auger recombination rate is given by $\left(C_{n} n+C_{p} p\right)(n p-$ $\left.n_{i}^{2}\right)$, where

$n$ electron density;

$p$ hole density;

$n_{i} \quad$ intrinsic carrier density.

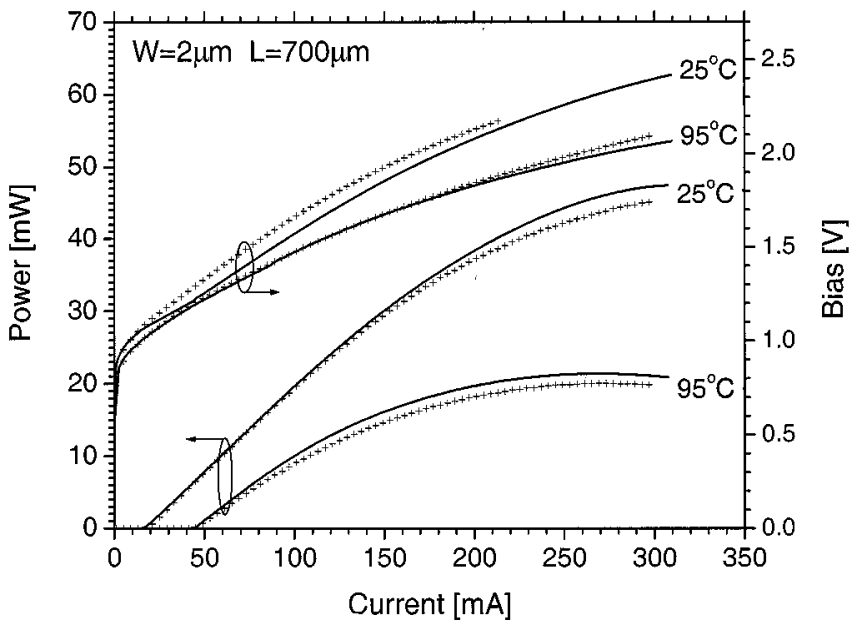

Fig. 6. Measured (+) and simulated (lines) $L-I$ and $I-V$ characteristics in CW operation at different ambient temperatures $(W=2 \mu \mathrm{m})$.

Both the Auger coefficients $C=C_{n}=C_{p}$ are assumed to be represented by a temperature dependent function

$$
C(T)=C_{0}\left(T_{0}\right) \times \exp \left[\frac{E_{A}}{k}\left(\frac{1}{T_{0}}-\frac{1}{T}\right)\right]
$$

where $k$ is the Boltzmann constant. The $L-I$ fit in Fig. 5 results in $C_{0}=5 \times 10^{-29} \mathrm{~cm}^{6} \mathrm{~s}^{-1}$ at $T_{0}=298 \mathrm{~K}$ and $E_{A}=100$ $\mathrm{meV}$. The activation energy $E_{A}$ is larger than that for $1.55-\mu \mathrm{m}$ InGaAsP/InP lasers [11] and smaller than that for $1.3-\mu \mathrm{m}$ InGaAsP/InP lasers [12].

The carrier mobility affects not only the $I-V$ characteristic, but also the Joule heat generated inside the device. The low mobility of holes dominates over the much higher electron mobility. CW measurements of $I-V$ characteristics are used to tune mobility parameters in the simulation. For all the semiconductors in our device, the temperature dependence of the hole mobility is given as

$$
\mu_{p}(T)=\mu_{\mathrm{po}}\left(\frac{300}{T}\right)^{2.2}
$$

where the room-temperature hole mobility $\mu_{\mathrm{po}}$ is used as a fit parameter to find agreement with the $I-V$ measurements. The resulting hole mobilities are listed in Table I. The measured top p-contact resistivity of $1.86 \times 10^{-6} \Omega \cdot \mathrm{cm}^{2}$ is also included in the simulation. Simultaneous agreement is obtained with the $I-V$ characteristic measured at two different temperatures (Fig. 6). Correct reproduction of the $I-V$ curve is a requirement for accurate calculation of the internal heat power generation.

Self-heating also depends on the thermal conductivity of each layer in our device, as well as on the thermal contact to the heat sink. Both are hard to determine accurately. The thermal conductivity values used are given in Table I. The simulated cross section of the laser includes $5 \mu \mathrm{m}$ of the InP substrate. The rest of the substrate, as well as the heat sink, are represented by a thermal resistance $R_{\text {th }}$ which is employed as the last remaining fit parameter in the $\mathrm{CW}$ simulation of $L-I$ characteristics (Fig. 6). For our unbonded device, this fit results in $R_{\mathrm{th}}=110 \mathrm{~K} / \mathrm{W}$. The same set of parameters gives good agreement with measurements between $T=25^{\circ} \mathrm{C}$ and $T=95^{\circ} \mathrm{C}$. 


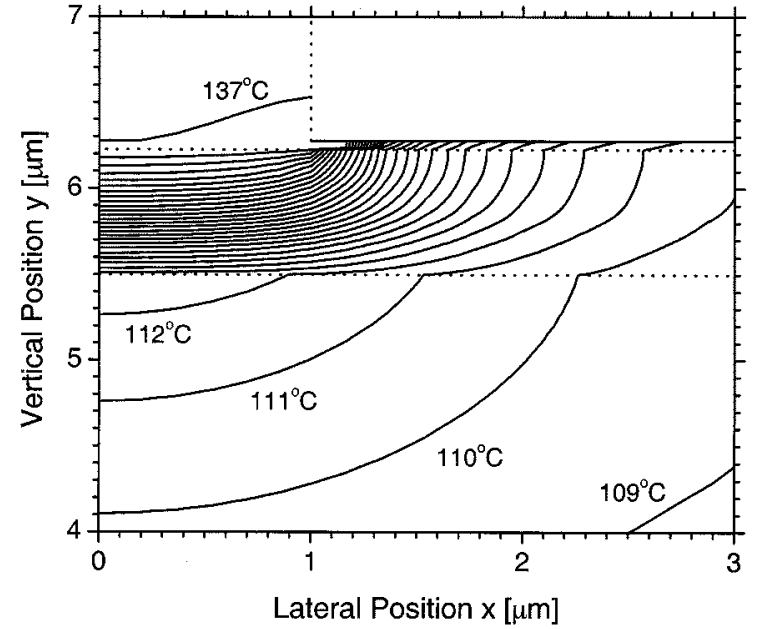

Fig. 7. 2-D temperature distribution at maximum power $\left(85^{\circ} \mathrm{C}\right.$ ambient temperature).

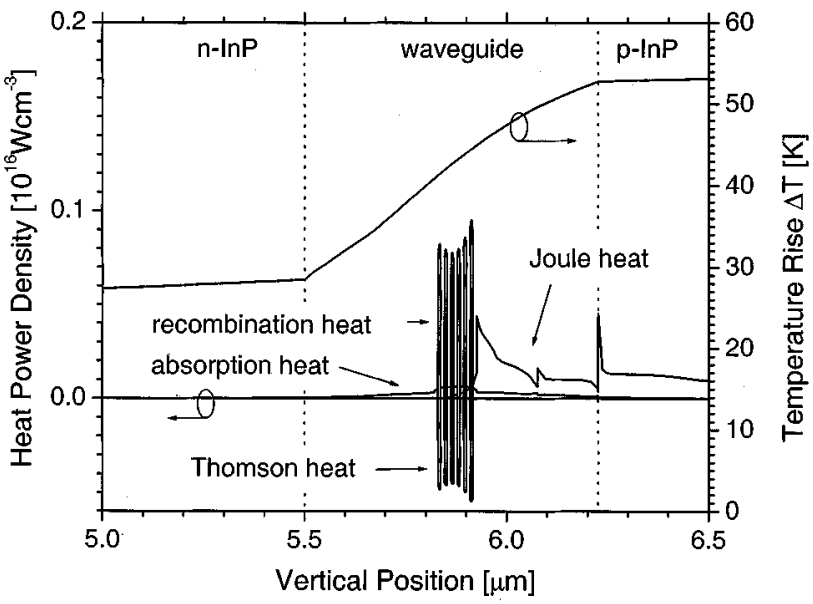

Fig. 8. Vertical heat power and temperature profile at maximum power.

The measured characteristic temperature of the threshold current is almost constant within this temperature range $\left(T_{o}=79\right.$ $\mathrm{K})$.

The above parameters are employed in the following to study the laser diode in high-temperature $\mathrm{CW}$ operation. The same ridge width of $W=2 \mu \mathrm{m}$ and the same cavity length of $L=$ $700 \mu \mathrm{m}$ will be assumed as with the laser used for parameter calibration.

\section{Performance Analysis}

In this section, we study the physical mechanisms that limit the maximum output power of the laser diode. Since these limitations are most serious at high ambient temperature, we assume a stage temperature of $85^{\circ} \mathrm{C}$, which is a typical maximum in telecommunications applications.

Fig. 7 plots the temperature distribution $T(x, y)$ at maximum power. The quantum-well temperature increase is about $\Delta T_{\mathrm{QW}}=45 \mathrm{~K}$ above the $85^{\circ} \mathrm{C}$ ambient temperature (Fig. 8). The main heat sources contributing to the temperature rise are plotted in Fig. 8. The strongest total contribution comes from Joule heating, which is mainly generated in the $p$-doped layers. Recombination heat is mainly generated in the quantum

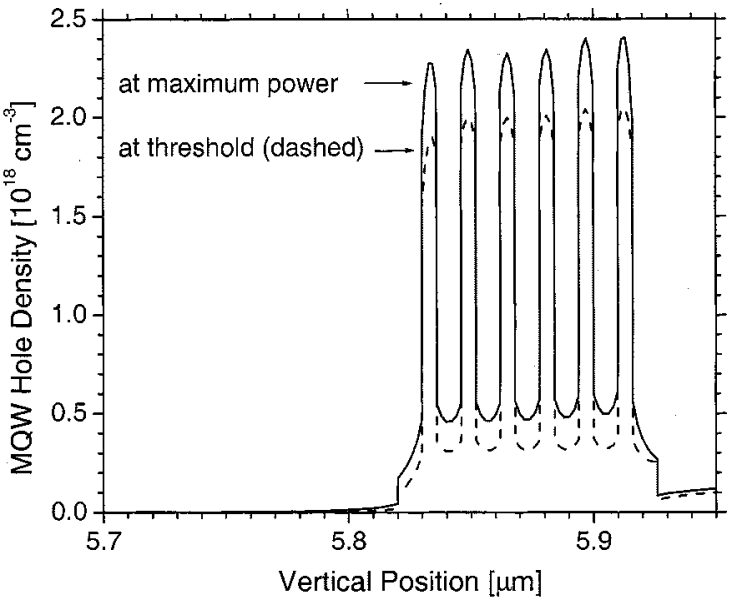

Fig. 9. MQW hole density at the laser axis for two power levels.

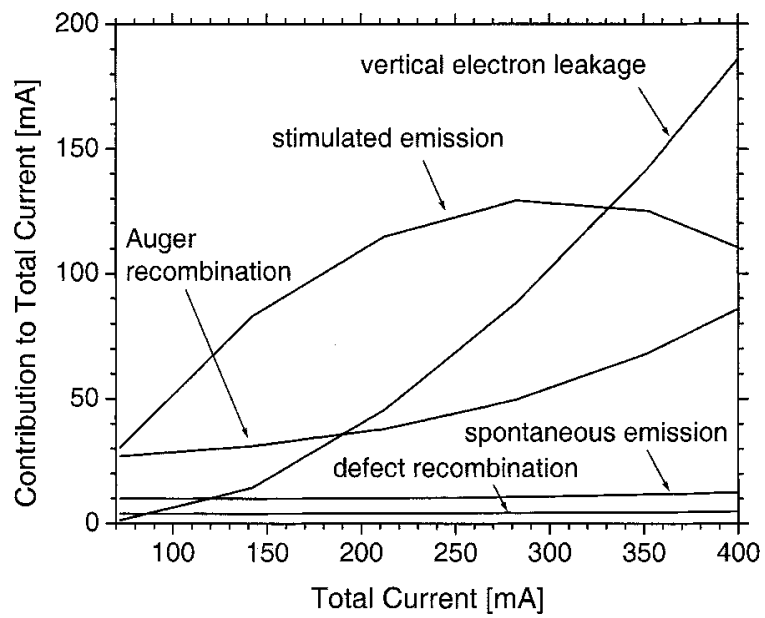

Fig. 10. Contributions to the total current.

wells, as well as negative Thomson heat which accounts for the capture and escape of quantum-well carriers (carrier escape requires energy and it removes heat from the lattice). Optical absorption heat is also strongest in the quantum wells due to absorption by holes.

The self-heating of the device on top of the high ambient temperature leads to a reduction of the quantum-well gain (cf. Fig. 2). This is caused by the wider Fermi spreading of carriers. As a consequence, the required carrier density within the quantum well rises (Fig. 9). Due to the small valence band offset, the hole distribution is almost uniform across the AlGaInAs quantum wells, which is in contrast to the strong nonuniformity calculated for InGaAsP/InP MQWs [13]. The increased quantum-well carrier density does not only maintain the threshold gain, but also results in significantly stronger Auger recombination. Spontaneous recombination and defect recombination also rise. All contributions to the laser current are compared in Fig. 10. The strongest increase occurs with the vertical electron leakage. This leakage current from the MQW active region into the $p$-InP ridge is illustrated in Fig. 11. Ideally, all electrons should recombine within the quantum wells and not penetrate the $p$-InP ridge. Any electron current on the $p$-side of the MQW constitutes vertical electron leakage. With 


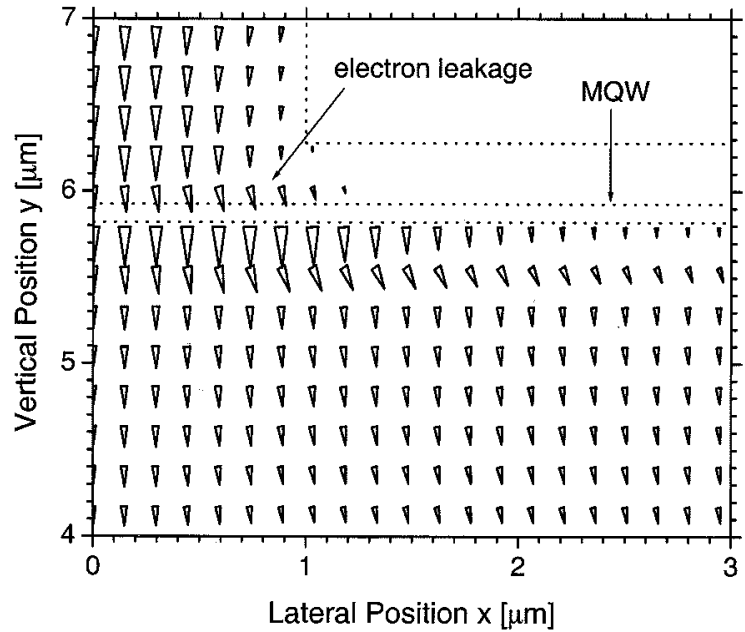

Fig. 11. Vector plot of the electron current density at maximum power (same direction as hole current).

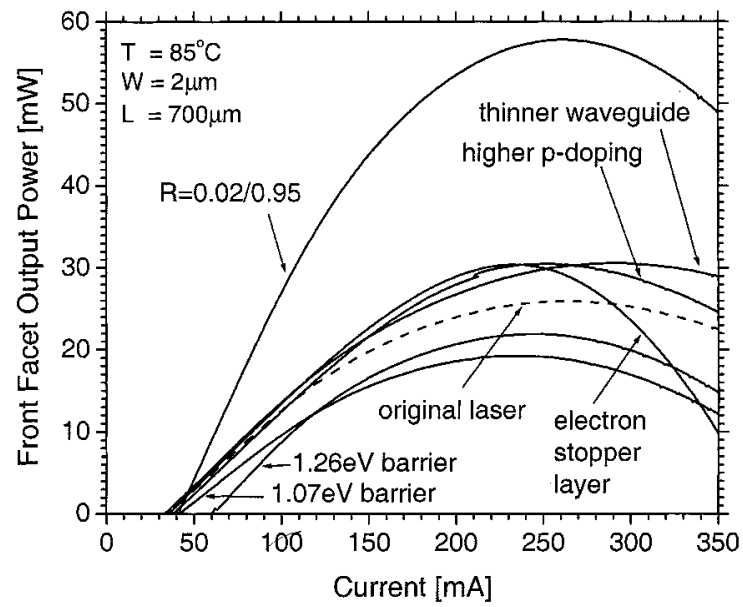

Fig. 12. $L-I$ characteristics for different design variations (dashed: original).

increasing current and temperature, vertical electron leakage becomes the dominating physical mechanism that reduces the QW carriers available for lasing. At maximum output power, vertical leakage amounts to about one third of the total current (Fig. 10).

\section{DESIGN VARIATION}

In this final section, several design variations are explored, aiming at an improvement of the maximum $\mathrm{CW}$ output power at $85^{\circ} \mathrm{C}$ ambient temperature. The resulting $L-I$ calculations are summarized in Fig. 12 and discussed in the following.

Higher power output from the laser's front facet can simply be achieved by increasing the back facet's reflectivity using appropriate coating. Anti-reflection coating of the front facet further improves the power. The output power is more than doubled by coating the facets with $R=0.02$ at the front and $R=0.95$ at the back (Fig. 12). This result is in agreement with previous experimental investigations [14].

All ternary and quaternary layers within the device exhibit a very low thermal conductivity (Table I). They are blocking the heat flux from the active region to the heat sink, which increases the MQW temperature significantly. Replacing some of

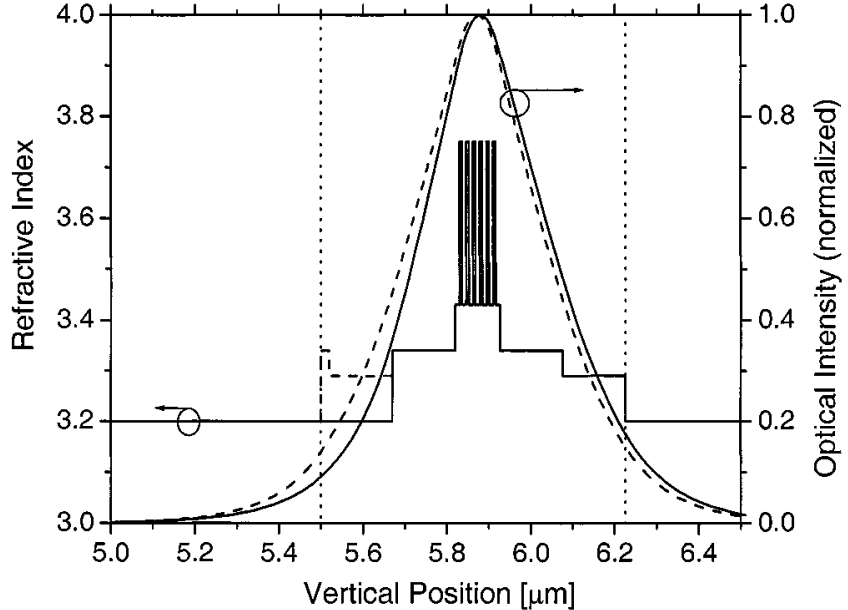

Fig. 13. Vertical profile of refractive index and optical mode with (solid) and without (dashed) waveguide modification.

the AlGaInAs layers by InP may reduce the self-heating and lead to a higher maximum output power. To simulate such a situation, two cladding layers on the $n$-side of the active region are replaced by InP. The effects on the refractive index profile and guided wave are shown in Fig. 13. The optical confinement factor is slightly improved from 0.083 to 0.087 . The simulated LI curve in Fig. 12 shows an increase in maximum power to $31 \mathrm{~mW}$. This is mainly related to a $10 \%$ reduction in the temperature rise in the active region.

The $p$ doping is kept low near the active region to reduce IVBA. However, low doping also reduces the AlInAs barrier height [15], increases the resistance, and enhances the self-heating. Thus, increasing the $p$ doping to $10^{18} \mathrm{~cm}^{-3}$ might be advantageous [16]. Indeed, Fig. 12 shows an improved maximum output power of $31 \mathrm{~mW}$. The internal optical loss rises from $\alpha_{i}=8.6$ to $9.8 \mathrm{~cm}^{-1}$. However, the active region self-heating at maximum power is only $\Delta T_{\mathrm{QW}}=32 \mathrm{~K}$, compared to $45 \mathrm{~K}$ with regular doping. This temperature reduction results in lower QW carrier density and less electron leakage.

AlInAs electron stopper layers have been proposed for improved high-temperature performance of AlGaInAs/InP lasers [17]. We include such a layer on top of the MQW (Fig. 14). The simulated $L-I$ characteristic in Fig. 12 exhibits an increased maximum output power of $31 \mathrm{~mA}$, mainly due to reduced electron leakage. However, the stopper layer adds electrical resistance to the device which causes stronger self-heating. This limits the power gain and it leads to a steeper roll-off in Fig. 12.

The MQW barrier bandgap of our structure is $1.165 \mathrm{eV}$ at room temperature, providing a large conduction band offset of $0.225 \mathrm{eV}$. Investigations on InGaAsP/InP lasers have shown that lowering this barrier may improve the laser performance [12]. Therefore, the bandgap of our unstrained MQW barrier layers is varied by changing the composition. Due to the similarity of the lattice constant in AlAs and GaAs, the $\mathrm{Ga}_{x} \mathrm{Al}_{y} \mathrm{In}_{1-x-y} \mathrm{As}$ strain is maintained by keeping $(1-x-y)$ constant [1]. Moreover, the bandgap energy changes almost linearly with the Al content. Table II gives the barrier parameters used in this comparison. The band offset does not change with temperature because the same function $E_{g}(T)$ is used for quantum well and barrier. The resulting LI curves are shown in Fig. 12. Surpris- 


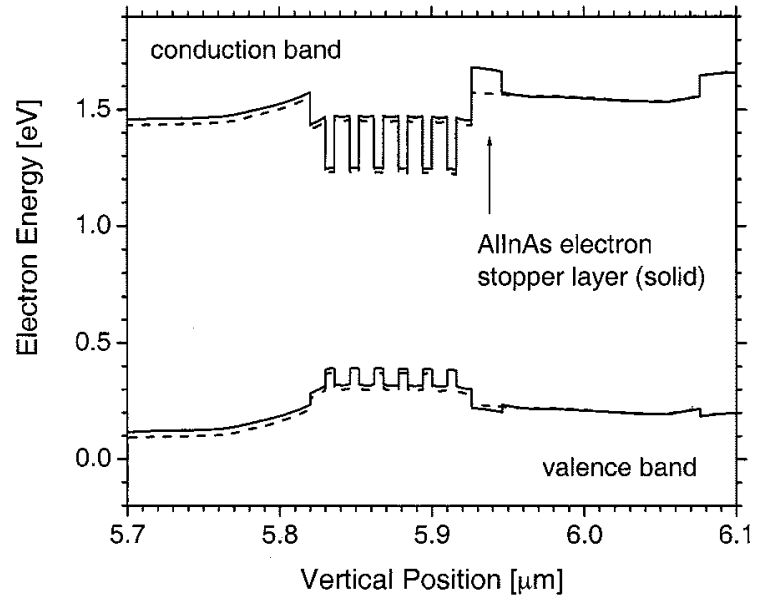

Fig. 14. Energy band diagram at threshold: with (solid) and without (dashed) electron stopper layer.

TABLE II

PARAMETERS OF THE $\operatorname{In}_{1-x-y} \mathrm{Ga}_{x} \mathrm{Al}_{y}$ As BARRIER LAYERS USED (ORIGINAL: $E_{g}=1.165 \mathrm{eV}$ )

\begin{tabular}{l|l|l|l|l|l}
\hline $\mathrm{x}$ & $\mathrm{y}$ & $\mathrm{E}_{\mathrm{g}} / \mathrm{eV}$ & $\Delta \mathrm{E}_{\mathrm{d}} / \mathrm{eV}$ & $\Delta \mathrm{E}_{\mathrm{v}} / \mathrm{eV}$ & $\mathrm{n}(1.3 \mu \mathrm{m})$ \\
\hline 0.21 & 0.27 & 1.165 & 0.225 & 0.091 & 3.43 \\
\hline 0.27 & 0.21 & 1.07 & 0.158 & 0.066 & 3.49 \\
\hline 0.15 & 0.33 & 1.27 & 0.292 & 0.116 & 3.39 \\
\hline
\end{tabular}

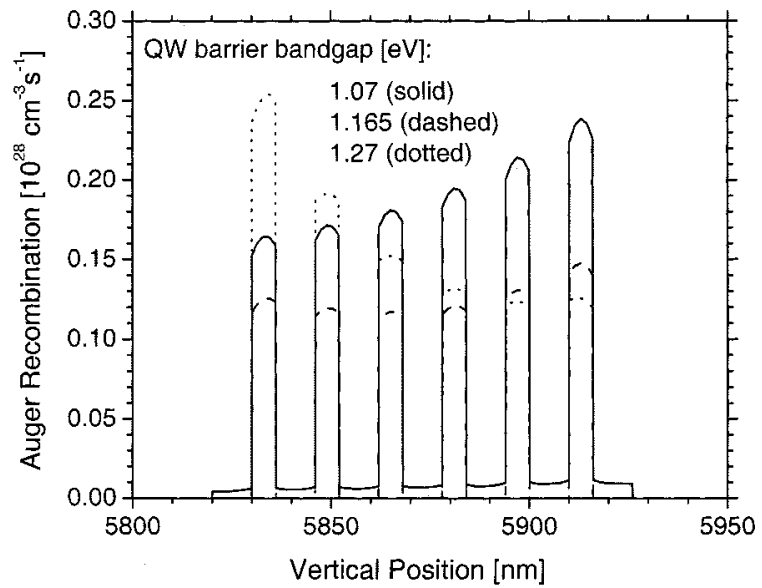

Fig. 15. Vertical profile of the Auger recombination rate with different MQW barrier bandgaps (dashed: original).

ingly, both the higher and the lower barrier bandgap deteriorate the LI performance. The reason for this can be understood from Fig. 15, which gives the vertical profile of the Auger recombination rate for all three cases. With a lower bandgap $(1.07 \mathrm{eV})$, the QW carrier density increases substantially toward the $p$ side because the electrons can move across the MQW more easily than the holes. With a higher bandgap $(1.27 \mathrm{eV})$, the QW carrier density increases toward the $n$ side because the holes can move across the MQW more easily than the electrons [18]. The total Auger recombination is enhanced in both cases, leading to higher threshold current and more self-heating. Obviously, the original barrier bandgap of $1.165 \mathrm{eV}$ represents a nearly optimum balance between the thermionic emission rates of electrons and holes so that the carrier distribution is almost uniform across the MQW and the Auger recombination is minimum.
Variations of the cavity length show that the present value of $L=700 \mu \mathrm{m}$ is nearly optimum. A wider ridge would reduce both the electrical and the thermal resistance; however, this option is not considered here due to the higher far-field aspect ratio and weaker suppression of higher order transversal modes.

\section{SUMMARY}

We show that the combination of laser measurements with advanced simulations allows for a detailed study of laser performance limitations. Key material parameters of the model are calibrated using experimental characteristics. The resulting agreement between measurements and simulations validates the laser model and it serves as a starting point for a detailed study of the internal physical mechanisms behind the output power roll-off at high temperatures. We show quantitatively that this roll-off is caused by an escalation of the electron leakage from the active region due to self-heating. The reduction of optical gain with temperature elevation is the triggering mechanism of this leakage escalation. Variations of the laser design in the simulations allow for further insight into the device physics. Modification of facet coating, $p$-doping, and waveguide design, as well as the inclusion of an electron-stopper layer, lead to a significant increase of the maximum output power.

\section{REFERENCES}

[1] C.-E. Zah, R. Bhat, B. N. Pathak, F. Favire, W. Lin, M. C. Wang, N. C. Andreadakis, D. M. Hwang, M. A. Koza, T.-P. Lee, Z. Wang, D. Darby, D. Flanders, and J. J. Hsieh, "High-performance uncooled 1.3- $\mu \mathrm{m}$ AlGaInAs/InP strained-layer quantum-well lasers for subscriber loop applications," IEEE J. Quantum Electron., vol. 30, pp. 511-523, Feb. 1994.

[2] J. Piprek, P. Abraham, and J. E. Bowers, "Self-consistent analysis of high-temperature effects on strained-layer multi-quantum well InGaAsP/InP lasers," IEEE J. Quantum Electron., vol. 36, pp. 366-374, Mar. 2000.

[3] A. J. SpringThorpe, T. Garanzotis, P. Paddon, G. Pakulski, and K. I. White, "Strained $1.3 \mu \mathrm{m} \mathrm{MQW} \mathrm{AlGaInAs} \mathrm{lasers} \mathrm{grown} \mathrm{by} \mathrm{digital} \mathrm{alloy}$ MBE," Electron. Lett., vol. 36, pp. 1031-1032, 2000.

[4] S. L. Chuang, "Efficient band structure calculations of strained quantum wells," Phys. Rev. B, vol. 43, pp. 9649-9661, 1991.

[5] J. Minch, S. H. Park, T. Keating, and S. L. Chuang, "Theory and experiment of InGaAsP and InGaAlAs long-wavelength strained quantum-well lasers," IEEE J. Quantum Electron., vol. 35, pp. 771-781, May 1999.

[6] M. J. Mondry, D. I. Babic, J. E. Bowers, and L. A. Coldren, "Refractive indexes of (Al, Ga, In)As epilayers on InP for optoelectronic applications," IEEE Photon. Technol. Lett., vol. 4, pp. 627-630, June 1992.

[7] S. Adachi, Physical Properties of III-V Semiconductor Compounds. New York: Wiley, 1992.

[8] M. Guden and J. Piprek, "Material parameters of quaternary III-V semiconductors for multilayer mirrors at $1.55 \mu \mathrm{m}$ wavelength," Model. Simul. Mater. Sci. Eng., vol. 4, pp. 349-357, 1996.

[9] J. Piprek, P. Abraham, and J. E. Bowers, "Cavity length effects on internal loss and quantum efficiency of multi-quantum-well lasers," IEEE J. Select. Topics Quantum Electron., vol. 5, pp. 643-647, May/June 1999.

[10] C. H. Henry, R. A. Logan, F. R. Merritt, and J. P. Luongo, "The effect of intervalence band absorption on the thermal behavior of InGaAsP lasers," IEEE J. Quantum Electron., vol. QE-19, pp. 947-952, 1983.

[11] S. Seki, W. W. Lui, and K. Yokoyama, "Explanation for the temperature insensitivity of the Auger recombination rates in $1.55 \mu \mathrm{m} \mathrm{InP-}$ based strained-layer quantum-well lasers," Appl. Phys. Lett., vol. 66, pp. 3093-3095, 1995.

[12] S. Mogg and J. Piprek, "Optimization of the barrier height in 1.3-micron InGaAsP multiple-quantum-well active regions for high temperature operation," in Proc. SPIE Physics and Simulation of Optoelectronic Devices, vol. 4283, 2001. paper 29. 
[13] J. Piprek, P. Abraham, and J. E. Bowers, "Carrier nonuniformity effects on the internal efficiency of multiquantum-well lasers," Appl. Phys. Lett., vol. 74, pp. 489-491, 1999.

[14] C.-C. Lin, M.-C. Wu, and W.-H. Wang, "Facet coating effects on 1.3 $\mu \mathrm{m}$ and $1.55 \mu \mathrm{m}$ strained multiple-quantum-well AlInGaAs/InP laser diodes," Proc. IEE-Optoelectronics, vol. 146, pp. 268-272, 1999.

[15] G. L. Belenky, C. L. Reynolds, D. V. Donetsky, G. E. Shtengel, M. S. Hybertsen, M. A. Alam, G. A. Baraff, R. K. Smith, R. F. Kazarinov, J. Winn, and L. E. Smith, "Role of $p$-doping profile and regrowth on the static characteristics of $1.3 \mu \mathrm{m}$ MQW InGaAsP-InP lasers: Experiment and modeling," IEEE J. Quantum Electron., vol. 35, pp. 1515-1520, Oct. 1999.

[16] J.-W. Pan, M.-H. Chen, and J.-I. Chyi, "High performance phosphorus-free $1.3 \mu \mathrm{m}$ AlGaInAs/InP MQW lasers," J. Cryst. Growth, vol. 201/202, pp. 932-926, 1999.

[17] R. F. Kazarinov and G. L. Belenky, "Novel design of AlGaInAs-InP lasers operating at $1.3 \mu \mathrm{m}$," IEEE J. Quantum Electron., vol. 31, pp. 423-426, May 1995.

[18] M. Grupen and K. Hess, "Simulation of carrier transport and nonlinearities in quantum-well laser diodes," IEEE J. Quantum Electron., vol. 34 pp. 120-140, Jan. 1998.

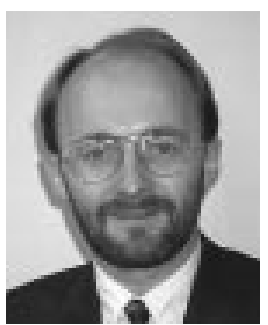

Joachim Piprek received the Ph.D. degree in solidstate physics from Humboldt University, Berlin, Germany, in 1986.

$\mathrm{He}$ is presently an Adjunct Associate Professor at the University of California at Santa Barbara. He has worked in industry and academia on the design and analysis of optoelectronic devices, and has given more than 40 invited talks, seminars, and short courses. He is the author of more than 50 papers in refereed journals, two book chapters, and an upcoming book on optoelectronic device simulation. His current research interests include long-wavelength vertical-cavity lasers, nitride semiconductor devices, and novel components for radio-frequency photonic links. He chairs the International Conference on Numerical Simulation of Semiconductor Optoelectronic Devices (NUSOD) and also serves as Guest Editor of special issues in this field.
J. Kenton White received the Ph.D. degree in 1999 from the University of Arizona, Tucson, for theoretical work on communicating with chaotic semiconductor lasers.

He joined the Scientific Staff at Nortel Networks Optical Components, Ottawa, ON, Canada, in 1999. Currently, he leads the modulated laser research and development for optical components. His research interests include exploiting laser dynamics for communication, AlGaInAs, and uncooled and buried heterostructure lasers. He has authored 35 refereed journal and conference articles.

Anthony J. SpringThorpe received the B.Sc. degree in physics in 1963 and the Ph.D. degree in solid-state physics in 1967 from the University of Sheffield, Sheffield, U.K.

This was followed by a three year post-doctoral fellowship at the University of Bath, Bath, U.K. He then joined Northern Electric R\&D Labs, Ottawa, ON, Canada, in October 1969, later to become Bell-Northern Research, and now Nortel Networks. As a member of Scientific Staff, he was responsible for liquid-phase epitaxial growth techniques for the preparation of III-V compound semiconductors, initially for visible light-emitting diodes, but subsequently for near infra-red lasers in the GaAs/AlGaAs and GaInAsP alloy systems. He designed and built the first Canadian MOCVD system in 1978. He was manager of epitaxy from 1978 to 1997 , and is now an independent contributor working as a New Materials Advisor in the High Performance Optical Components Division, Nortel Networks. He currently holds over 25 patents on various aspects of III-V compound semiconductor epitaxy and devices and, during his professional life, has contributed to more than 160 publications and presentations on materials and devices.

Dr. SpringThorpe has been on the organizing and program committees of numerous international conferences on III-V compounds and related devices over the last 30 years. He was awarded the W. Lash Miller Prize of the Electrochemical Society for his work on the development of III-V compound materials and devices and the Nortel Presidents' Prize for technology cascade, both in 1977. He is currently an active member of the program committees of the International and North American MBE conferences. 